\title{
Interphase effect on the interfacial debonding behavior in
}

\section{short-fiber-reinforced rubber composites}

\author{
Xiaoming $\mathrm{Yu}^{1, \mathrm{a}}$, Boqin $\mathrm{Gu}^{1, \mathrm{~b}^{*}}$, Bin Zhang ${ }^{2, \mathrm{c}}$, Lili Chen ${ }^{1, \mathrm{~d}}$ andJiahui Tao ${ }^{1, \mathrm{e}}$ \\ ${ }^{1}$ School of Mechanical and Power Engineering, Nanjing Tech University, Nanjing, PR China \\ ${ }^{2} S$ chool of Mechanical Engineering, Changshu Institute of Technology, Changshu, PR China \\ adryxm@njtech.edu.cn, bbqgu@njtech.edu.cn, drzhbin@163.com, dIlchen@njtech.edu.cn, \\ tjh18751903358@163.com
}

\section{Keywords: Fiber reinforced composites, Stress transfer, Interphase, Debonding}

Abstract. The interfacial debonding behavior of short-fiber-reinforced composites (SFRC) can be an interesting subject for many investigators. In this paper, the effects of interphase properties on the interfacial debonding behavior of SFRC are investigated. Based on the shear lag model, the initial strain of the interfacial debonding is derived. The results of analytical model show that the interfacial debonding behavior strongly depends on the interphase elastic modulus and thickness and suitable interphase parameters could prevent the onset of the interfacial debonding.

\section{Introduction}

Fiber-reinforced composites have been widely used for various kinds of structures because of its high specific strength, stiffness, and robust endurance. The reinforcement of rubber with fibers is one of these types [1]. Interphase, or interfacial zone, in fiber-reinforced composite materials is the thin layers between the fiber and the matrix. Although small in thickness, interphase can significantly affect the overall mechanical properties of the fiber or particle reinforced composites [2]. For example, a stiff interphase can transfer stress much more rapidly than a soft interphase [3]. In order to form an interphase, Hayes et al. [4] applied an epoxy resin with known properties to the surface of untreated reinforcing fibers. The effect of interphase thickness, by applying multiple coats of the resins, and the effect of the interphase properties, by varying the coating resins were studied. Ryu et al. [5] investigated the effects of multiple coatings with various combinations of rubber and bonding agent by measuring the tensile and fatigue properties of short-fiber-reinforced rubber.

A number of micromechanics models have been developed to predict the stress transfer, among which the shear-lag model [6,7] and the finite element analysis [8,9] are the most widely used. Yuan et al. [10] presented an interphase layer mode to describe the interphase of composites and analyzed the effects of the interphase thickness and interfacial shear strength on the peak load. Ghavami et al. [11] developed a finite difference formulation to study the creep behavior of short-fiber-reinforced metal matrix composites under a given axial tensile load. The shear stress based debonding model is capable of accurately predicting the debonding parameter. Jiang et al. [12] used a simple theory and a special frictional contact-element method to analyze stress transfer from matrix to fiber, the shear strength and the tensile strength of fiber/matrix interface are used to judge the slipping or debonding of interface. Lu et al. [13] presented a theoretical model is for the interfacial debonding and frictional pull-out of a single elastic fiber which is coated with a plastic coating from an elastic matrix. Parametric studies on two kinds of composite systems, SiC 
fiber-glass matrix composites and carbon fiber-epoxy matrix composites, showed that increasing the yield stress and decreasing the elastic modulus of the coating improved the debonding stress at any debonding length.

The interfacial debonding is a common damage type in fiber-reinforced composites. The study of interphase is taken into consideration more and more by researchers, however there are few reports on the effects of interphase properties about the interfacial debonding behavior. Based on the shear-lag model, this manuscript derives the equation of the critical strain of interfacial debonding and analyzes the influences of the interphase modulus and thickness on interfacial debonding length of SFRC.

\section{Shear-lag model}

In short-fiber or whisker composites, loads are not directly applied on the fibers but are applied to the matrix and transferred to the fibers through the cylindrical surface of the fiber. In order to model the composites, it is necessary to extract a unit cell out of it. Here, it is assumed that the fibers are regularly dispersed in the matrix along the axis of extrusion [14]. The cylindrical unit cell depicted in Fig. 1 has been used by many researchers to model a short-fiber composite. This cell contains a single fiber and adjoining interphase and matrix materials. The subscript $\mathrm{f}, \mathrm{i}$ and $\mathrm{m}$ are used to stand for fiber, interphase and matrix, respectively. The axial direction is $x$-axis and radial direction is $r$-axis. The radius of fiber and matrix are $r_{\mathrm{f}}$ and $r_{\mathrm{m}}$, respectively, and the interphase is a circular cylindrical shell of inner radius $r_{\mathrm{f}}$ and outer radius $r_{\mathrm{i}}$. The interphase thickness $t=r_{\mathrm{i}}-r_{\mathrm{f}}$. The length of fiber is $2 l$.

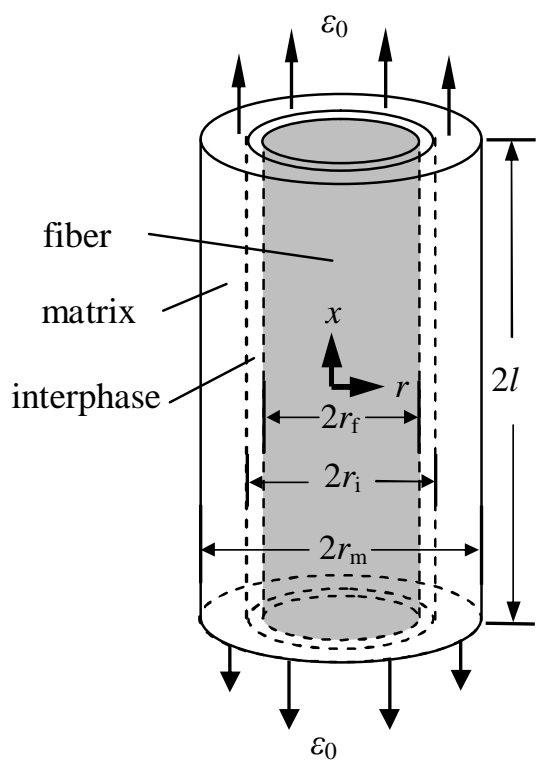

Fig. 1 Micromechanical model of SFRC.
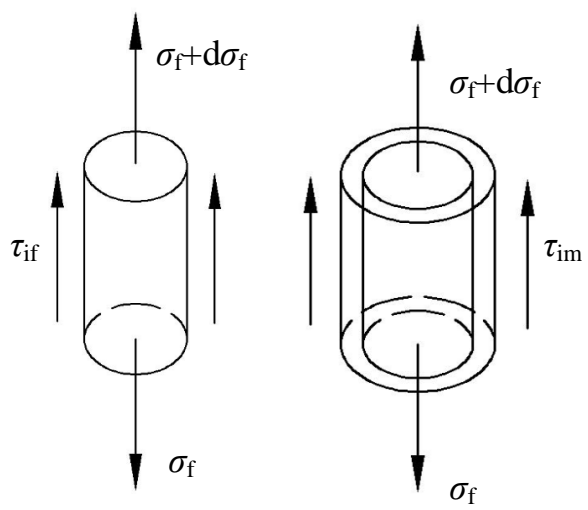

The micromechanical stress transfer in unit cell is shown in Fig. 2. The equilibrium equations are as follows

$$
\begin{aligned}
& \pi r_{\mathrm{f}}^{2}\left[\sigma_{\mathrm{f}}+\mathrm{d} \sigma_{\mathrm{f}}-\sigma_{\mathrm{f}}\right]+2 \pi r_{\mathrm{f}} \mathrm{d} x \tau_{\mathrm{if}}=0 \\
& \pi r_{\mathrm{f}}^{2}\left[\sigma_{\mathrm{f}}+\mathrm{d} \sigma_{\mathrm{f}}-\sigma_{\mathrm{f}}\right]+2 \pi r_{\mathrm{i}} \mathrm{d} x \tau_{\mathrm{im}}=0
\end{aligned}
$$


From equations (1) and (2), we have

$$
\frac{\mathrm{d} \sigma_{\mathrm{f}}}{\mathrm{d} x}=-\frac{2 \tau_{\mathrm{if}}}{r_{\mathrm{f}}}=-\frac{2 \tau_{\mathrm{im}} r_{\mathrm{i}}}{r_{\mathrm{f}}^{2}} .
$$

where $\sigma_{\mathrm{f}}$ is the tensile stress in the fiber, $\tau_{\text {if }}$ is the shear stress on $r=r_{\mathrm{f}}, \tau_{\mathrm{im}}$ is the shear stress on $r=$ $r_{\mathrm{i}}$.

According to the similar derivation progress of reference [7], we have the differential equation of the tensile stress in the fiber

$$
\frac{\mathrm{d}^{2} \sigma_{\mathrm{f}}}{\mathrm{d} x^{2}}-\frac{\alpha^{2}}{r_{\mathrm{f}}^{2}} \sigma_{\mathrm{f}}=-\frac{\alpha^{2}}{r_{\mathrm{f}}^{2}} E_{\mathrm{f}} \varepsilon_{0}
$$

where

$$
\alpha^{2}=\frac{2 G_{\mathrm{i}} G_{\mathrm{m}} r_{\mathrm{i}}}{E_{\mathrm{f}} r_{\mathrm{f}} G_{\mathrm{i}} \ln \left(r_{\mathrm{m}} / r_{\mathrm{i}}\right)+E_{\mathrm{f}} r_{\mathrm{i}} G_{\mathrm{m}} \ln \left(r_{\mathrm{i}} / r_{\mathrm{f}}\right)}
$$

Applying the following boundary conditions

$$
\begin{cases}x=-l, & \sigma_{\mathrm{f}}=0 \\ x=l, & \sigma_{\mathrm{f}}=0\end{cases}
$$

The tensile stress in the fiber and the shear stress on the interface can be expressed as follows

$$
\begin{aligned}
\sigma_{\mathrm{f}} & =E_{\mathrm{f}} \varepsilon_{0}-E_{\mathrm{f}} \varepsilon_{0} \cosh \left(\alpha x / r_{\mathrm{f}}\right) / \cosh (\alpha s) \\
\tau_{\mathrm{if}} & =\frac{1}{2} E_{\mathrm{f}} \varepsilon_{0} \alpha \sinh \left(\alpha x / r_{\mathrm{f}}\right) / \cosh (\alpha s)
\end{aligned}
$$

where $E_{\mathrm{f}}$ is the elastic modulus of fiber. $G_{\mathrm{i}}$ and $G_{\mathrm{m}}$ are the shear modulus of the interphase and the matrix, respectively. $\varepsilon_{0}$ is the axial tensile strain at $r=r_{\mathrm{m}}$. The aspect ratio of fiber $s$ can be written as

$$
s=l / r_{\mathrm{f}}
$$

When the shear stress at the interface reaches a critical value $\tau_{\mathrm{a}}$, the friction sliding will happen. Ordinarily, the frictional resistance is less than $\tau_{\mathrm{a}}$. From equation (8), the maximal shear stress is

$$
\tau_{\max }=\frac{1}{2} E_{\mathrm{f}} \varepsilon_{0} \alpha \tanh (\alpha s)
$$

When the friction sliding happen, the applied strain is $\varepsilon_{0 \mathrm{~s}}$, according to the sliding condition: $\tau_{\max }$ $=\tau_{\mathrm{a}}$, the critical strain of interfacial debonding can be expressed as

$$
\varepsilon_{0 \mathrm{~s}}=2 \tau_{\mathrm{a}} \operatorname{cth}(\alpha s) / \alpha E_{\mathrm{f}}
$$

Defining the length of the sliding region is $\mathrm{ml} . \mathrm{m}$ is dimensionless parameter which depends on the applied load. When $x=l(1-m)$ and $\sigma_{\mathrm{f}}=\sigma_{\mathrm{fi}}, \sigma_{\mathrm{fi}}$ is the normal stress in the fiber of the sliding region. So, the stress distribution of the bonding region in the fiber is

$$
\sigma_{\mathrm{fc}}=E_{\mathrm{f}} \varepsilon_{0}+\left(\sigma_{\mathrm{fi}}-E_{\mathrm{f}} \varepsilon_{0}\right) \cosh \left(\alpha x / r_{\mathrm{f}}\right) / \cosh (\alpha \bar{s})
$$

where $\bar{s}=s(1-\mathrm{m})$, which is called the equivalent aspect ratio.

Then, differentiating Equation (12) with respect to $x$, we have 
$\frac{\mathrm{d} \sigma_{\mathrm{fc}}}{\mathrm{d} x}=\frac{\alpha}{r_{\mathrm{f}}}\left(\sigma_{\mathrm{fi}}-E_{\mathrm{f}} \varepsilon_{0}\right) \sinh \left(\alpha x / r_{\mathrm{f}}\right) / \cosh (\alpha \bar{s})$

When $x \geq l(1-m), \frac{\mathrm{d} \sigma_{\mathrm{fc}}}{\mathrm{d} x}=-2 \frac{\tau_{\mathrm{a}}}{r_{\mathrm{f}}}$. Then combining equations (13), we have

$\sigma_{\mathrm{fi}}-E_{\mathrm{f}} \varepsilon_{0}=-\frac{2 \tau_{\mathrm{a}}}{\alpha} \operatorname{cth}(\alpha \bar{s})$

Normal compressive stress on the interface $\sigma_{\mathrm{r}}=\lambda_{\mathrm{m}} E_{\mathrm{m}} \varepsilon_{0}$, where $\lambda_{\mathrm{m}}$ is the Poisson's ratio of rubber. In the sliding region, the shear stress at the interface is $\mu \sigma_{\mathrm{r}}$. On the basis of equations (3), we have

$$
\sigma_{\mathrm{fc}}=\frac{2 \mu \sigma_{\mathrm{rt}}}{r_{\mathrm{f}}}(l-x)
$$

When $x=l(1-m)$, the normal stress at the interface is:

$\sigma_{\mathrm{fi}}=2 \mu \sigma_{\mathrm{rt}} \mathrm{ms}$

From equations (13), the relative length of the sliding region is

$$
\mathrm{m}=\frac{E_{\mathrm{f}} \varepsilon_{0}-2 \tau_{\mathrm{a}} \operatorname{cth}(\alpha \bar{s}) / \alpha}{2 \mu s \sigma_{\mathrm{rt}}}
$$

\section{Results and discussion}

The material properties and dimension of fiber, interphase and rubber matrix used in the calculation of stress profile by analytical model is shown in Table 1. The interfacial shear strength between fiber and interphase is assumed to $8 \mathrm{MPa}$ [2]. On the basis of equation (11), the critical strain of interfacial debonding is $8.08 \%$.

The failure mechanisms of fiber-reinforced composites include fiber interfacial debonding, matrix cracking and fiber breakage. For short-fiber-reinforced rubber composites (SFRC), because the tensile stress of the fiber is much less than the tensile strength of fiber and rubber possesses the property of large deformation, the main failure behavior of SFRC is interfacial debonding.

\begin{tabular}{cccc}
\hline Property & Fiber & Interphase & Rubber \\
\hline Elastic modulus [MPa] & 2000 & 11.58 & 20 \\
Poisson's ratio & 0.22 & 0.35 & 0.47 \\
Diameter [mm] & 0.028 & 0.030 & 0.056 \\
Length of the fiber [mm] & 1 & 1 & 1 \\
\hline
\end{tabular}

Table 1 Material properties and dimension of fiber, interphase and rubber

Fig. 3 shows the tensile stress distribution on the fiber interface at the strain of $8 \%$. It can be seen that the tensile stress along fiber reaches to the maximum at the fiber midpoint, and they are equal to zero at the fiber end. However, the interfacial shear stress equal zero at the fiber midpoint, and it reaches to the maximum at the end of the fiber where the interfacial debonding will happen. When the strain achieves $8.30 \%$, the interfacial debonding happens and the debonding length is $0.07 \mathrm{~mm}$ (equal to $14 \%$ of $l$ ). It can be seen from Fig. 4 that the interfacial shear stress at fiber end declines sharply and then keeps constant. The reason is that, once interfacial debonding happens, the stress transfers from the matrix to the fiber through the friction mainly. The fiber tensile stress at the fiber 
end becomes linear distribution.

The interphase elastic modulus and thickness determine the critical strain of interfacial debonding. Different interphase thickness $(t=0.5 \mu \mathrm{m}, 1 \mu \mathrm{m}, 2 \mu \mathrm{m})$ and different interphase elastic modulus $\left(E_{\mathrm{i}}=1 \sim 50 \mathrm{MPa}\right)$ are considered to investigated debonding behavior of SFRC. Fig. 5 shows that the critical strain of interfacial debonding $\left(\varepsilon_{0 \mathrm{~s}}\right)$ decreases as the interphase elastic modulus $E_{\mathrm{i}}$ increases. $\varepsilon_{0 \mathrm{~s}}$ descends quickly when $E_{\mathrm{i}}$ is less than $12 \mathrm{MPa}$ and it descends slowly when $E_{\mathrm{i}}$ is greater than $12 \mathrm{MPa}$. It can be seen that $\varepsilon_{0 \mathrm{~s}}$ trends to a constant value with high interphase elastic modulus. It is interesting that the $\varepsilon_{0 \mathrm{~s}}$ is equal to $8 \%$ with different interphase thickness when $E_{\mathrm{i}}$ is 12 MPa. Fig. 5 also shows the critical strain of interfacial debonding increases with the interphase thickness when $E_{\mathrm{i}}$ is less than $12 \mathrm{MPa}$ and it decreases with the interphase thickness when $E_{\mathrm{i}}$ is larger than $12 \mathrm{MPa}$.

Fig. 6 shows the effects of interphase on the debonding length of SFRC. The loads were applied to the matrix, and the strain is $8 \%$. The interface begins to debond when the interphase elastic modulus reach to $15 \mathrm{MPa}$. The interfacial debonding length increases with the increase of interphase elastic modulus and interphase thickness. Therefore, the low interphase elastic modulus and thin interphase thickness can prevent the development of the interfacial debonding.

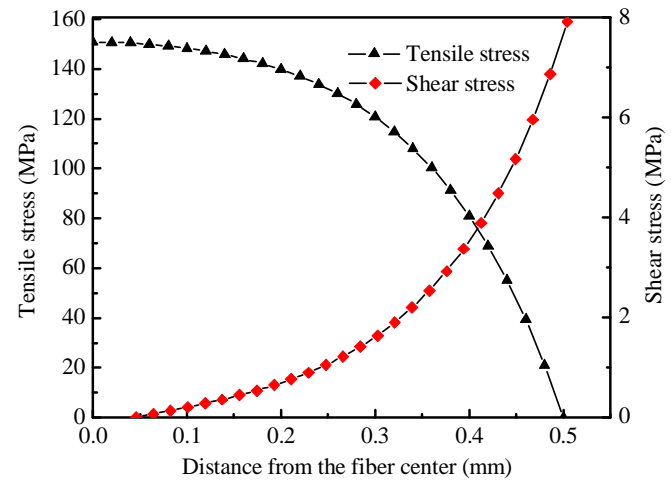

Fig. 3 The tensile and shear stress distributions at the strain of $8 \%$.

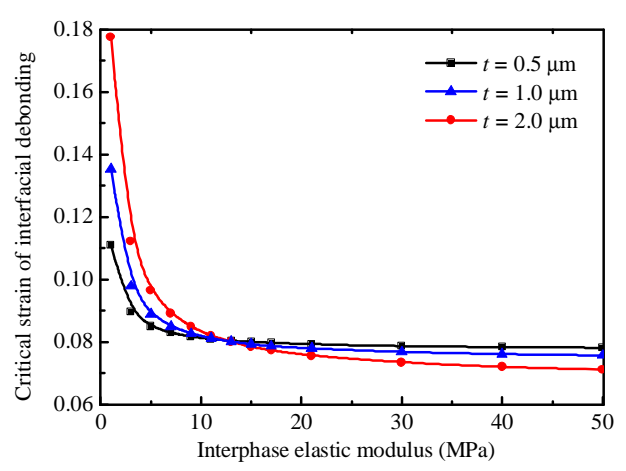

Fig. 5 The critical strain of interfacial debonding Fig. 6 The effects of interphase elastic modulus varies as the interphase elastic modulus.

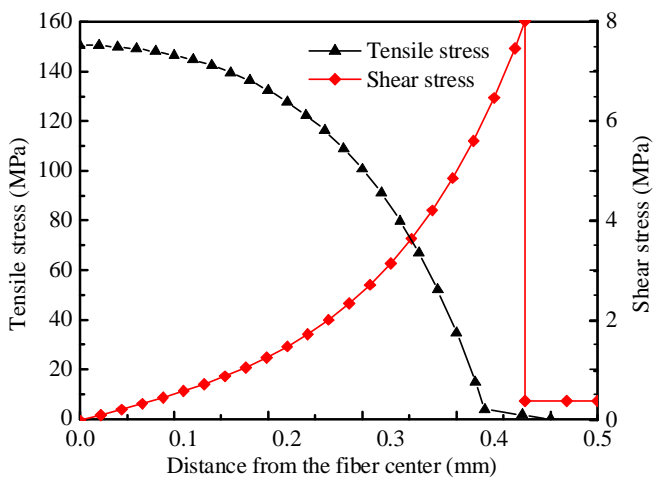

Fig. 4 The tensile and shear stress distributions at the strain of $8.3 \%$.

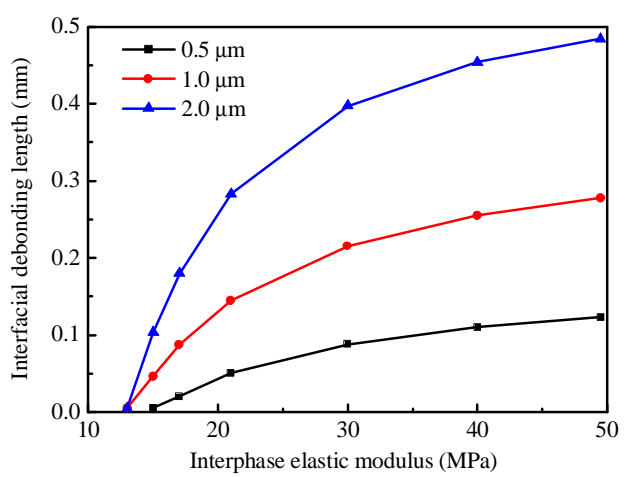
and thickness on the interfacial debonding length.

\section{Conclusions}

Based on shear lag model, the initial strain and relative length of the interfacial debonding is derived. The load-carrying characteristics and stress profiles in SFRC subjected to an applied tensile load was obtained. The results show that the interfacial shear stress reaches to the maximum at the end of the fiber where the interfacial debonding will happen. The influences of interphase elastic modulus and thickness on interfacial debonding behavior are investigated. The results show that the 
low interphase elastic modulus and thin interphase thickness can prevent the development of the interfacial debonding.

\section{Acknowledgements}

This project is supported by National Natural Science Foundation of China (Grant No. 51375223) and by Jiangsu Province Ordinary University Graduate Research and Innovation Foundation (Grant No. KYZZ15_0230).

\section{References}

[1] S.R. Ryu, D.J. Lee, Effects of interphase on the mechanical properties of short-fiber reiforced rubber, Compos. Interface. 13 (2006) 173-187.

[2] B. Zhang, B.Q. Gu, X.M. Yu. Failure behavior of resorcinol formaldehyde latex coated aramid short fiber reinforced rubber sealing under transverse tension, J. Appl. Polym. Sci. (132) 2015 doi: 10.1002/APP.41672.

[3] A.C. Johnson, S.A. Hayes, F.R. Jones, An improved model including plasticity for the prediction of the stress in fibres with an interface/interphase region, Compos. Part A. 36 (2005) 263-271.

[4] S.A. Hayes, R. Lane, F.R. Jones, Fibre/matrix stress transfer through a discrete interphase. Part 1: single-fibre model composites, Compos. Part A. 32 (2001) 379-389.

[5] S.R. Ryu, D.J. Lee, Effects of Interphase and Short Fiber on Puncture and Burst Properties of Short-fiber Reinforced Chloroprene Rubber, J. Elastom. Plast. 42 (2010) 181-197.

[6] M. Mondali, A. Abedian, A. Ghavami, A new analytical shear-lag based model for prediction of the steady state creep deformations of some short fiber composites, Mater. Design. 30 (2009) 1075-1084.

[7] X. Wang, C. Liu, Z. Tang, Y. Wang, Analysis on the process of stress transferring and characterization on the property of "interphase" in three-phase single-fiber composites, J. Reinf. Plast. Compos. 33 (2014) 1388-1402.

[8] B. Zhang, B.Q. Gu, The Effect of Interphase Modulus and Thickness on Stress Transfer of Short-Fiber-Reinforced Composites, Appl. Mech. Mat. 55 (2011) 303-307.

[9] X.M. Yu, B.Q. Gu, B. Zhang, Effects of short fiber tip geometry and inhomogeneous interphase on the stress distribution of rubber matrix sealing composites, J. Appl. Polym. Sci. 132 (2015) doi:10.1002/app.41638.

[10]M.N. Yuan, Y.Q. Yang, H.J. Luo, Evaluation of interfacial properties in SiC fiber reinforced titanium matrix composites using an improved finite element model, Mater. Charact. 59 (2008) 1684-1689.

[11]A. Ghavami, A. Abedian, M. Mondali, Finite difference solution of steady state creep deformations in a short fiber composite in presence of fiber/matrix debonding, Mater. Design. 31 (2010) 2616-2624. 
[12]X.Y. Jiang, Q. Gao, Stress-transfer analysis for fibre/matrix interfaces in short-fibre-reinforced composites, Compos. Sci. Technol. 61 (2001) 1359-1366.

[13] G.Y. Lu, Y.W. Mai, Effect of plastic coating on fibre-matrix interface debonding, J. Mater. Sci. 30 (1995) 5872-5878.

[14]T. Morimoto, T. Yamaoka, H. Lilholt, M. Taya, Second stage creep of SiC whiskers/6061 aluminum composite at 573 K, J. Eng. Mater. Technol. 110 (1988) 70-76. 